\title{
VEGF-C and VEGF-D overexpression is more common in left-sided and well-differentiated colon adenocarcinoma
}

\author{
MARIUSZ SZAJEWSKI ${ }^{1,2}$, WIESŁAW JANUSZ KRUSZEWSKI ${ }^{1,2}$, JOANNA LAKOMY ${ }^{3}$, MACIEJ CIESIELSKI ${ }^{1,2}$, \\ KRZYSZTOF KAWECKI ${ }^{1}$, JERZY JANKUN ${ }^{4,5}$, TOMASZ BUCZEK ${ }^{1}$ and JAROSŁAW SZEFEL ${ }^{1,2}$ \\ ${ }^{1}$ Department of Surgical Oncology, Gdynia Oncology Centre, PCK's Maritime Hospital in Gdynia, 81-519 Gdynia; \\ ${ }^{2}$ Department of Propaedeutics of Oncology, Faculty of Health Sciences, Medical University of Gdańsk, 81-519 Gdynia; \\ ${ }^{3}$ Department of Pathology, Medical University of Gdańsk, 80-214 Gdańsk; ${ }^{4}$ Department of Clinical Nutrition, \\ Medical University of Gdańsk, 80-211 Gdańsk, Poland; ${ }^{5}$ Urology Research Center, Department of Urology, \\ The University of Toledo, Health Science Campus, Toledo, OH 43614, USA
}

Received August 21, 2013; Accepted October 8, 2013

DOI: $10.3892 / o r .2013 .2821$

\begin{abstract}
Tumour vessel network formation, including blood and lymph vessels, is a major step involved in the process of carcinogenesis. The discovery of vascular growth factors has led to a better understanding of tumour biology, thus, creating new possibilities for cancer treatment that targets angiogenesis within tumour-associated stroma, including therapy for colon cancer patients. The present study evaluated the relationships between increased expression of lymphangiogenic factors (VEGF-C and VEGF-D) and vessel density in the tumoursurrounding stroma, patient survival and other standard prognostic factors. The expression of VEGF-C and VEGF-D and vessel density were immunohistochemically assessed in 114 primary tumour specimens from colon adenocarcinoma patients after surgical resection between January 1, 2003 and December 31, 2008. Concomittant overexpression of VEGF-C and VEGF-D was found in $51(44.7 \%)$ colon tumours and low expression was observed in $63(55.3 \%)$ cases. Mean vessel density was 52.87/field. A significant correlation was found between the expression of factors influencing lymph vessel growth and increased vessel density in the tumour-surrounding stroma $(\mathrm{p}=0.03)$. A relationship between lymphangiogenic factor overexpression and left-sided tumour location was also found $(\mathrm{p}=0.00002)$. Overexpression of these factors was likely to occur in well-differentiated tumours $(p=0.003)$. No association between patient survival and the expression levels of lymphangiogenic factors was observed. The study results indicate that the overexpression of lymphangiogenic factors tends to be associated with tumours of favourable prognosis,
\end{abstract}

Correspondence to: Dr Mariusz Szajewski, Department of Surgical Oncology, Gdynia Oncology Centre, PCK's Maritime Hospital in Gdynia, Powstania Styczniowego 1, 81-519 Gdynia, Poland

E-mail:mszaj@gumed.edu.pl

Key words: VEGF-C, VEGF-D, lymphangiogenesis, colon cancer i.e. well-differentiated and those localized in the left-side of the colon.

\section{Introduction}

Colorectal cancer is one of the most common malignancies worldwide. High morbidity and unsatisfactory therapeutic efficacy warrant further research in cancer cell biology. The progression of this disease occurs together with the participation of the blood and lymph vascular network generated in the tumour from pre-existing vessels (1-3). Vascular endothelial growth factor (VEGF), also known as vascular permeability factor (VPF), is the main mediator of angiogenesis (4). Increased expression of VEGF promotes proliferation and migration of arterial, venous and lymphatic endothelial cells, thus playing a key role in both angiogenesis and lymphangiogenesis $(5,6)$. VEGF is a protein belonging to the platelet-derived growth factor family. The VEGF subfamily includes VEGF-A, VEGF-B, VEGF-C, VEGF-D and placenta growth factor (PlGF), which display distinct biological activities $(7,8)$. VEGF is synthesised and secreted by fibroblasts, endothelial cells, tumour cells, macrophages, lymphocytes and megakaryocytes (9-11). VEGF exerts its action by binding to glycoprotein receptors VEGFR-1 (Flt-1), VEGFR-2 (Flk-1/ KDR) and VEGFR-3 (Flt-4), and by forming signalling complexes (12-14). The predominant function in stimulating proliferation, growth and maturation of lymphatic endothelial cells is attributed to VEGF-C and VEGF-D. Both of these factors bind and activate VEGF-3 and VEGF-2 receptors. In mature tissue, VEGFR-s is expressed only in lymphatic endothelial cells, whereas VEGFR-2 is present on both lymphatic and vascular endothelial cell surfaces (15). Their expression is most commonly assessed by immunohistochemistry (16).

Research on vascular growth factors, their receptors and angiogenesis and lymphangiogenic markers has provided the basis for the development of targeted cancer therapy $(17,18)$.

The present study investigated the overexpression of lymphangiogenic factors (VEGF-C and VEGF-D) in primary colon tumours and its correlation with vessel density in the 
tumour-associated stroma, prognosis and conventional clinicopathological prognostic factors.

\section{Materials and methods}

Paraffin-embedded primary tumour specimens obtained from 114 consecutive patients who underwent surgery for colon cancer between January 1, 2003 and December 31, 2008, were included in the present study. In each case, the tumour location was limited to the colon. All patients were treated with surgical resection at the General Surgery Unit and since 2006, at the Department of Oncological Surgery, Gdynia Centre of Oncology, Maritime Hospital in Gdynia, Poland.

In each case, the local range of the primary tumour resection met the criteria for the radical procedure, regardless of the cancer grade. None of the patients received any adjuvant therapy. Patients with synchronous and metachronous colorectal carcinoma or with cancers in other organs were excluded from the present study. Women comprised $54.4 \%(n=62)$ of the study participants and men, $45.6 \%(n=52)$. Participants ranged in age from 32 to 90 years (mean 69.9 years; median 70 years). Patients were followed up for a period of 80 months. All deaths during the observation period were caused by malignancy. The mean survival time was 37.8 months, with a median of 37 months.

Table I presents the patient clinical data. The recorded age refers to the age at the time of the surgery. Histological categorisation of each tumour was based on the WHO classification system (19). All cases included in the study were diagnosed as adenocarcinoma. An adenocarcinoma mucinosum was considered poorly differentiated (G3). The colon was divided into the right $(\mathrm{R})$ and left $(\mathrm{L})$ sides. The right side included the caecum, ascending colon, hepatic flexure and the proximal two-thirds of the transverse colon. The left side included the distal third of the transverse colon, splenic flexure, descending colon and sigmoid colon. Tumours were classified according to the pTNM system (20).

Immunohistochemistry. Immunohistochemical staining was performed on tissue sections fixed with $4 \%$ formaldehyde and embedded in low melting point paraffin. The paraffinembedded tissue blocks were cut into $5-\mu \mathrm{m}$ thick sections with a sledge microtome and transferred to silane-coated slides.

The immunochemical staining was performed in 114 resected specimens of colon tumours and in 10 normal colon mucosa samples as a control. After deparaffinisation and rehydration with distilled water, the sections were subjected to heat-induced antigen retrieval with a citrate buffer $(\mathrm{pH} 6.0)$ at $99^{\circ} \mathrm{C}$ for $40 \mathrm{~min}$ in a water bath. Endogenous peroxidase was then blocked by $3 \%$ hydrogen peroxide for $10 \mathrm{~min}$.

The following primary antibodies were used. i) CD34: monoclonal mouse anti-human (Dako, cat. no. M 7165); antibody dilution 1:50; incubation at room temperature for $30 \mathrm{~min}$; detection for $30 \mathrm{~min}$ using the EnVision system (Dako, cat. no. K 4011). ii) VEGF-D (C-18): goat polyclonal IgG (Santa Cruz Biotechnology, Inc., cat. no. 7602); antibody dilution 1:200; incubation overnight at $4^{\circ} \mathrm{C}$ (in refrigerator); detection for $30 \mathrm{~min}$ using the LSAB system (Dako, cat. no. K 0690). iii) VEGF-C (C-20): goat polyclonal IgG (Santa Cruz Biotechnology, Inc., cat. no. 1881); antibody dilution 1:200;
Table I. Clinicopathological characteristics of 114 patients with colon adenocarcinoma.

\begin{tabular}{lr} 
Parameter & No. of p \\
\hline Age, (years) & \\
Mean age, (70 years) & 60 \\
$>70$ & 54 \\
$\leq 70$ & \\
Gender & 62 \\
Female (F) & 52 \\
Male (M) & \\
Tumour localization in colon & 63 \\
Right-sided (R) & 51 \\
Left-sided (L) & 6 \\
Histological grade of tumour & 88 \\
G1 & 20 \\
G2 & \\
G3 &
\end{tabular}

Spread of primary tumour (T)

$\mathrm{T} 1$

$\mathrm{T} 2$

T3

$\mathrm{T} 4(\mathrm{a}+\mathrm{b})$

$14(10+4)$

Regional lymph nodes (N)

N0

68

$\mathrm{N} 1(\mathrm{a}+\mathrm{b}+\mathrm{c})$

$28(11+13+4)$

$\mathrm{N} 2(\mathrm{a}+\mathrm{b})$

$18(11+7)$

Distant metastasis (M)

M0

100

M1 (a+b)

$14(11+3)$

Tumour stage

I

II $(\mathrm{A}+\mathrm{B}+\mathrm{C})$

7

III $(\mathrm{A}+\mathrm{B}+\mathrm{C})$

$57(53+4+0)$

IV $(\mathrm{A}+\mathrm{B})$

$36(1+32+3)$

$14(11+3)$

incubation overnight at $4^{\circ} \mathrm{C}$ (in refrigerator); detection for 30 min using the LSAB system (Dako, cat. no. K 0690).

The sections were visualized by incubation with 3,3'-diaminobenzidine (DAB) for $10 \mathrm{~min}$ at room temperature, then counterstained with Mayer's haematoxylin and mounted with Canada balsam. Between particular stages of the procedure, the sections were rinsed with phosphate-buffered saline (PBS) twice for $5 \mathrm{~min}$. Microscopic examination was performed using an Olympus CX41.

Cytoplasmic expression of VEGF-C and/or VEGF-D in tumour cells was scored on a scale from 0 to 3 , according to the intensity of the staining: no staining, 0 ; weak staining, 1+; moderate staining, $2+$, and strong staining, $3+$.

In addition, the percentage of cytoplasmic staining of the tumour cells was scored on a scale of 0 to 5: $0 \%$ staining, 0 ; 0 to $>1 \%$ staining $1 ; 1$ to $>10 \%$ staining, $2 ; 10$ to $>33 \%$ staining, 3; 33 to $>66 \%$ staining, 4 and $>66 \%$ staining, 5 . 

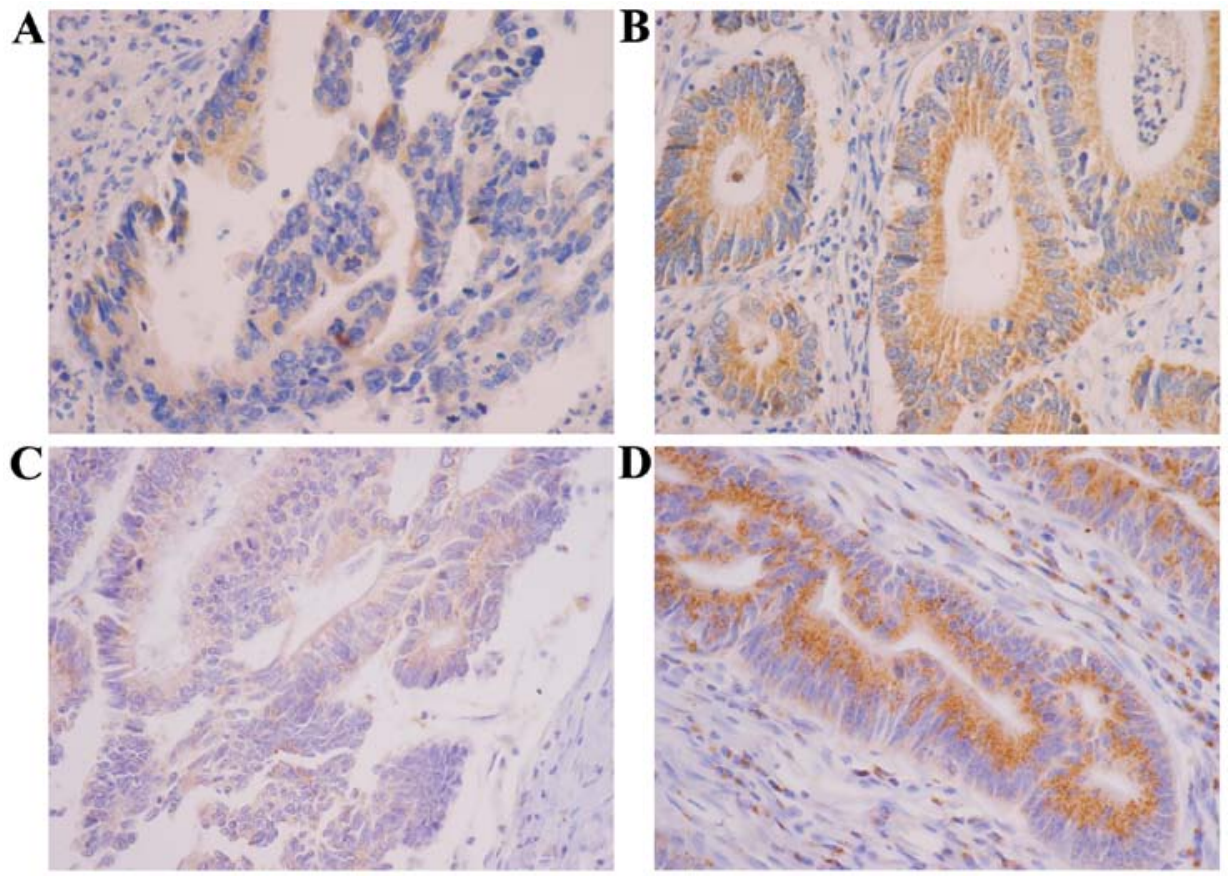

Figure 1. Microscopic images of colon tumours. (A) Negative staining and (B) positive staining for VEGF-C; (C) negative staining and (D) positive staining for VEGF-D.

For overall expression of VEGF-C and/or VEGF-D, a total score (TS) was calculated by combining staining intensity (a) and proportion of positively stained cells (b), as follows:

$$
\mathrm{TS}=\frac{\mathrm{a}+\mathrm{b}}{8} .
$$

The equation was based on the scoring system developed and used by Allred et al for the evaluation of oestrogen and progesterone receptor expression in breast carcinoma $(21,22)$.

If TS was $5 / 8$ or greater $(5 / 8=$ median) and $7 / 8$ or greater $(7 / 8=$ median $)$, the expression was considered positive for VEGF-C and VEGF-D, respectively.

Vascular density was assessed by utilizing CD34 monoclonal antibodies according to the method previously described by Weidner et al (23).

The immunochemical findings were correlated to: a) patient survival time (in months), b) median age, c) gender, d) tumour localization, i.e. right-sided vs. left-sided, e) spread of primary tumour $(\mathrm{pT})$, regional lymph node involvement $(\mathrm{pN})$, and occurrence of distant metastasis $(\mathrm{pM}), \mathrm{f})$ tumour stage according to the pTNM system (I-IV) and g) tumour histological grade (G1-G3).

Statistical analysis. Analysis was carried out using the statistical software package, Statistica v.9.1 (StatSoft Company, http://www.statsoft.com/company). The Mann-Whitney $\mathrm{U}$ test was used to evaluate the relationship between vessel density in the tumour-surrounding stroma and the expression of lymphangiogenic factors. The correlation between the expression of lymphangiogenic factors and clinicopathological characteristics was assessed using the Pearson's Chi-square test. Yates' correction was applied if the expected count was $<5$. Survival analysis was performed using the Kaplan-Meier method and the log-rank test was used to compare differences between survival times in groups. The statistical significance level was set at $\mathrm{p}<0.05$.

\section{Results}

Fig. 1 presents the microscopic images of immunohistochemical staining for the lymphangiogenic factors analysed.

Primary tumours positive for VEGF-C and VEGF-D (TS equal to or higher than the median) were assigned to group A. The tumours with a TS lower than the median, for at least one of the factors analysed, were assigned to group B. Group A consisted of $51(44.7 \%)$ patients with overexpression of lymphangiogenic factors. The remaining 63 (55.3\%) patients, with low lymphangiogenic factor expression in tumour cells, constituted group B. Mean vessel density/field was 52.87 (median, 45.75; range, 13-175; standard deviation, 28.05). In normal colon specimens obtained from healthy controls $(\mathrm{n}=10)$, TS values for both VEGF-C and VEGF-D expression were lower than the median in the tumour group. The expression of VEGF-C and VEGF-D in the tumour-surrounding stroma in 114 patients is shown in Table II.

For statistical analysis purposes, clinicopathological characteristics were pooled and compared as follows: T1+T2 vs. $\mathrm{T} 3+\mathrm{T} 4, \mathrm{G} 1+\mathrm{G} 2$ vs. G3; tumour stage, I+II vs. III+IV.

A positive correlation was found between the expression of lymph vessel growth factors and increased vascular density in tumour-associated stroma ( $\mathrm{p}=0.03$; Fig. 2). In Table III, the relationship between lymph vessel growth factor expression in groups A and B with the analysed clinicopathological parameters is shown. The overexpression of lymphangiogenic factors was found to be associated with left-sided tumour location $(\mathrm{p}=0.00002)$ and with tumour histological grade $(\mathrm{p}=0.003)$. Over $88 \%$ of poorly differentiated tumours did not express lymphangiogenic factors and $94 \%$ of them were right-sided 
Table II. Summary of lymphangiogenic factor expression analysis in 114 tumour specimens.

Cytoplasmic expression of lymphangiogenic factors in tumour-associated stroma

Tumour specimens n $(\%)$

\section{VEGF-C}

Positive tumours

Negative tumours

VEGF-D

Positive tumours

Negative tumours

Table III. Relationship between expression of the lymphangiogenic factors (group A vs. B) and clinicopathological characteristics using the Pearson's Chi-square test.

\begin{tabular}{lcc}
\hline Parameter & $\begin{array}{c}\text { Pearson's } \chi^{2} \text { value } \\
\text { (group A vs. B) }\end{array}$ & $\begin{array}{c}\text { P-value } \\
\text { (group A vs. B) }\end{array}$ \\
\hline
\end{tabular}

\begin{tabular}{l}
\hline Age, (years) \\
$>70$ \\
$\leq 70$ \\
Gender \\
Female \\
Male \\
Tumour localization \\
Right-sided \\
Left-sided
\end{tabular}

Spread of primary

tumour

0.00002

Yates' correction

ns

$\mathrm{pT} 1+\mathrm{pT} 2$

pT3+pT4

Regional lymph nodes

0.04

ns

$\mathrm{N}(+)$

N0

Metastasis

M1

M0

Tumour stage

I+II

III+IV

Tumour grade

8.78

0.003

$\mathrm{G} 1+\mathrm{G} 2$

G3

ns, not significant.

(L vs. R, p=0.0004). In $79 \%$ of metastatic (M1) tumours, in both groups $\mathrm{A}$ and $\mathrm{B}$, lymphangiogenic factor overexpression was not observed $(\mathrm{p}=0.06)$. Furthermore, no association was

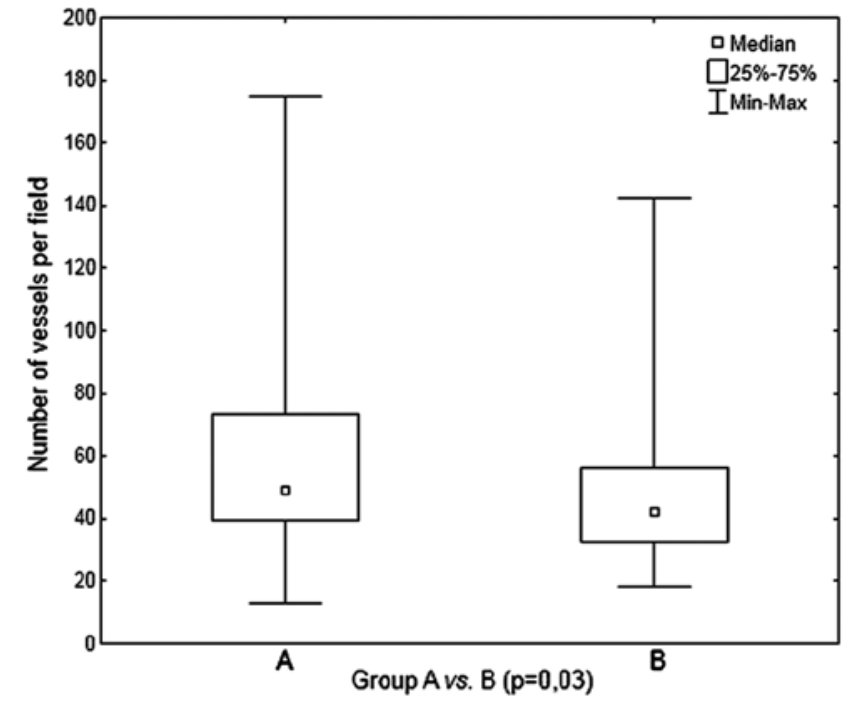

Figure 2. Correlation between lymphangiogenic factor expression in group A vs. B and vessel density in tumour-surrounding stroma.

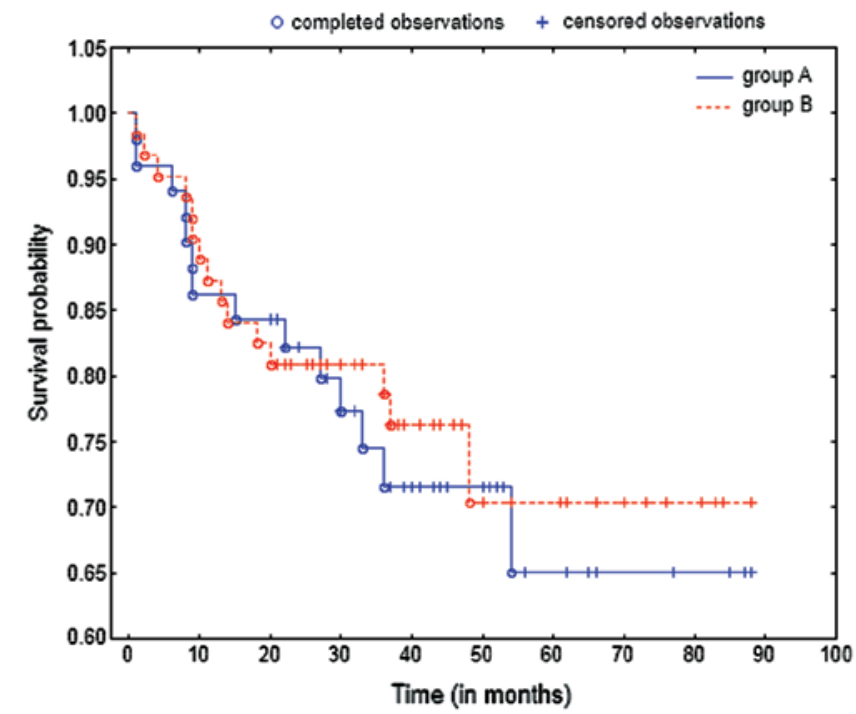

Figure 3. Kaplan-Meier survival curves for groups A and B ( $\mathrm{p}=0.7$, log-rank test).

found between lymphangiogenic factor expression (VEGF-C and/or VEGF-D) in groups A or B and prognosis ( $\mathrm{p}=0.7, \log$ rank test; Fig. 3).

\section{Discussion}

Most commonly, VEGF-C positivity is defined when it is expressed in over $10 \%$ of tumour cells, as assessed by immunohistochemical staining. With reference to this threshold, VEGF-C expression in colorectal carcinoma was found to range between 47 and 67\% (24-26). A number of authors has combined the intensity of immunohistochemical staining for VEGF-C with the proportion of positively stained cells to examine VEGF-C expression. According to this approach, a $68 \%$ VEGF-C frequency in primary colon carcinoma has been reported (27). This approach was also used in the present study. 
Based on the immunohistochemical staining, positive expression for VEGF-D ranged from 30 to even $100 \%$ in the colorectal tumours. Similarly to VEGF-C, over $10 \%$ of tumour cell staining was considered to indicate VEGF-D positivity. It is important to note that not all researchers have precisely defined VEGF-D positivity (28). In our study, VEGF-C and VEGF-D expression was scored according to the Allred et al system, which was previously used for the evaluation of hormone receptors in breast carcinoma (21). In contrast to the other authors, we combined data for VEGF-C and VEGF-D expression and pooled patients with high lymphangiogenic factor expression in tumour cells into group $\mathrm{A}$ and patients with low expression into group B. Thus, approximately $45 \%$ of colon adenocarcinoma cases were classified into group A. According to the defined positivity criteria, $57 \%$ of tumours showed VEGF-C expression and $61 \%$ were positive for VEGF-D. Furthermore, the frequency of expression for each lymphangiogenic factor in the primary tumour cells exceeded $50 \%$. Similar results have been reported by other authors, regardless of the scoring system used for VEGF-C and VEGF-D expression.

CD34 is a vascular endothelial cell marker (29). In one study, the expression of CD34 was reported to be specific only for lymphatic vessels (30). When our study was conducted, more specific markers (such as podoplanin) were not widely used. Hence, to assess vascular density within primary colon tumours, we used the CD34 antigen. Assuming that CD34 is a marker for endothelial cells of the microvessels in the lymphatic network, our evaluation of vessel density may be related to the marked microvessel network of the lymphatic system. Thus, indirectly, we deduced that group A consisted of patients with higher vessel density compared to group B. Our results revealed a positive correlation between increased vessel density and lymphangiogenic factor overexpression in primary colon cancer. This is in agreement with the observations of other authors, who used different methods and found a similar association between the overexpression of lymphangiogenic factors and increased intra-tumour vessel density (31). We have not found reports in which a similar scoring system has been used for lymphangiogenesis evaluation. Our method combines two different characteristics of lymphangiogenesis. One of them is lymph vessel density and the other is the outcome of the expression of two lymphangiogenic factors (VEGF-C and VEGF-D), determined from the equation described in the Materials and methods section.

As we used an original approach, it is difficult to directly compare our results to the findings of other authors. The majority reported an overexpression of both VEGF-C and VEGF-D as being independently associated with poor prognosis in patients with colon carcinoma (32). We were unable to confirm these results in our study. Although we did not demonstrate the relationship between analysed factors and prognosis, we demonstrated a significantly higher expression of lymphangiogenic factors in left-sided colon tumours. The results of the previous studies indicated that tumours with this localisation were associated with more favourable prognoses than right-sided cancers (33-35). We also demonstrated that tumours with lymphangiogenic factor overexpression were more commonly well-differentiated and associated with a more favourable prognosis. It was significantly more common that poorly differentiated colon tumours were right-sided. A longer asymptomatic period during cancer progression or a different expression of genes associated with tumour development (compared to left-sided tumours) may account for the poor prognosis associated with right-sided tumours $(36,37)$. In colon carcinomas, an increase in the proportion of poorly differentiated tumours together with disease progression has been observed (38-40). Thus, the prevalence of poorly differentiated tumours increases with time among slowly progressing right-sided cancers. It cannot be ruled out that progressive accumulation of gene mutations and phenotypic changes within primary tumour cells may lead to inhibition of cancer cell differentiation and that decreased lymphangiogenic factor expression accounts for the morphological result. This is one of the possible hypotheses justifying the observed relationship between lymphangiogenic factor overexpression and well-differentiated, left-sided colon tumours.

In conclusion, overexpression of lymphangiogenic factors in colon adenocarcinoma was associated with increased vessel density in tumour-surrounding stroma. Left-sided tumour localisation within the colon promotes overexpression of lymph vessel growth factors. Moreover, overexpression of lymphangiogenic factors is more common in well-differentiated tumours.

\section{References}

1. Carmeliet P and Jain RK: Angiogenesis in cancer and other diseases. Nature 407: 249-257, 2000.

2. Jain RK: Molecular regulation of vessel maturation. Nat Med 9: 685-693, 2003.

3. Bergers $G$ and Benjamin LE: Tumorigenesis and the angiogenic switch. Nat Rev Cancer 3: 401-410, 2003.

4. Dvorak HF: Vascular permeability factor/vascular endothelial growth factor: a critical cytokine in tumor angiogenesis and a potential target for diagnosis and therapy. J Clin Oncol 20: 4368-4380, 2002.

5. Ferrara N, Gerber HP and LeCouter J: The biology of VEGF and its receptors. Nat Med 9: 669-676, 2003.

6. Ferrara $\mathrm{N}$ and Davis-Smyth T: The biology of vascular endothelial growth factor. Endocr Rev 18: 4-25, 1997.

7. Shibuya M: Structure and function of VEGF/VEGF-receptor system involved in angiogenesis. Cell Struct Funct 26: 25-35, 2001.

8. Nagy JA, Vasile E, Feng D, et al: Vascular permeability factor/ vascular endothelial growth factor induces lymphangiogenesis as well as angiogenesis. J Exp Med 196: 1497-1506, 2002.

9. Bellamy WT, Richter L, Frutiger Y and Grogan TM: Expression of vascular endothelial growth factor and its receptors in hematopoietic malignancies. Cancer Res 59: 728-733, 1999.

10. Salgado R, Benoy I, Bogers J, et al: Platelets and vascular endothelial growth factor (VEGF): a morphological and functional study. Angiogenesis 4: 37-43, 2001.

11. Wang ES, Teruya-Feldstein J, Wu Y, Zhu Z, Hicklin DJ and Moore MA: Targeting autocrine and paracrine VEGF receptor pathways inhibits human lymphoma xenografts in vivo. Blood 104: 2893-2902, 2004

12. Pajusola K, Aprelikova O, Korhonen J, et al: FLT4 receptor tyrosine kinase contains seven immunoglobulin-like loops and is expressed in multiple human tissues and cell lines. Cancer Res 52: 5738-5743, 1992.

13. Kaipainen A, Korhonen J, Mustonen T, et al: Expression of the fms-like tyrosine kinase 4 gene becomes restricted to lymphatic endothelium during development. Proc Natl Acad Sci USA 92: 3566-3570, 1995.

14. Cao Y, Chen H, Zhou L, et al: Heterodimers of placenta growth factor/vascular endothelial growth factor. Endothelial activity, tumor cell expression, and high affinity binding to Flk-1/KDR. J Biol Chem 271: 3154-3162, 1996.

15. Jussila L and Alitalo K: Vascular growth factors and lymphangiogenesis. Physiol Rev 82: 673-700, 2002. 
16. Rinderknecht $\mathrm{M}$ and Detmar M: Molecular mechanisms of lymph-node metastasis. In: Lymphangiogenesis in Cancer Metastasis. Stacker S and Achen M (eds). Vol 13. Springer, pp55-82, 2009.

17. Nagahashi M, Ramachandran S, Rashid OM and Takabe K: Lymphangiogenesis: a new player in cancer progression. World J Gastroenterol 16: 4003-4012, 2010.

18. Tarallo V, Vesci L, Capasso O, et al: A placental growth factor variant unable to recognize vascular endothelial growth factor (VEGF) receptor-1 inhibits VEGF-dependent tumor angiogenesis via heterodimerization. Cancer Res 70: 1804-1813, 2010.

19. Bosman FT, Carneiro F, Hruban RH and Theise ND (eds): WHO Classification of Tumors of the Digestive System. International Agency for Research on Cancer, Lyon, 2010.

20. Edge SB, Byrd DR and Compton CC (eds): WHO classification of Tumours of the Digestive System. World Health Organization, Geneva, 2010.

21. Allred DC, Harvey JM, Berardo M and Clark GM: Prognostic and predictive factors in breast cancer by immunohistochemical analysis. Mod Pathol 11: 155-168, 1998.

22. Arihiro K, Oda M, Ogawa K, et al: Comparison of evaluations of hormone receptors in breast carcinoma by image-analysis using three automated immunohistochemical stainings. Exp Ther Med 1: 927-932, 2010.

23. Weidner N, Carroll PR, Flax J, Blumenfeld W and Folkman J: Tumor angiogenesis correlates with metastasis in invasive prostate carcinoma. Am J Pathol 143: 401-409, 1993.

24. Onogawa S, Kitadai Y, Tanaka S, Kuwai T, Kimura S and Chayama K: Expression of VEGF-C and VEGF-D at the invasive edge correlates with lymph node metastasis and prognosis of patients with colorectal carcinoma. Cancer Sci 95: 32-39, 2004.

25. Jia YT, Li ZX, He YT, Liang W, Yang HC and Ma HJ: Expression of vascular endothelial growth factor-C and the relationship between lymphangiogenesis and lymphatic metastasis in colorectal cancer. World J Gastroenterol 10: 3261-3263, 2004.

26. Akagi K, Ikeda Y, Miyazaki M, et al: Vascular endothelial growth factor-C (VEGF-C) expression in human colorectal cancer tissues. Br J Cancer 83: 887-891, 2000.

27. Soumaoro LT, Uetake H, Takagi Y, et al: Coexpression of VEGF-C and Cox-2 in human colorectal cancer and its association with lymph node metastasis. Dis Colon Rectum 49: 392-398, 2006.

28. White JD, Hewett PW, Kosuge D, et al: Vascular endothelial growth factor-D expression is an independent prognostic marker for survival in colorectal carcinoma. Cancer Res 62: 1669-1675, 2002.
29. Krause DS, Fackler MJ, Civin CI and May WS: CD34: structure, biology, and clinical utility. Blood 87: 1-13, 1996.

30. Fiedler U, Christian S, Koidl S, et al: The sialomucin CD34 is a marker of lymphatic endothelial cells in human tumors. Am J Pathol 168: 1045-1053, 2006.

31. Li X, Liu B, Xiao J, Yuan Y, Ma J and Zhang Y: Roles of VEGF-C and Smad4 in the lymphangiogenesis, lymphatic metastasis, and prognosis in colon cancer. J Gastrointest Surg 15: 2001-2010, 2011.

32. Hu WG, Li JW, Feng B, et al: Vascular endothelial growth factors $\mathrm{C}$ and $\mathrm{D}$ represent novel prognostic markers in colorectal carcinoma using quantitative image analysis. Eur Surg Res 39: 229-238, 2007.

33. Benedix F, Kube R, Meyer F, et al: Comparison of 17,641 patients with right- and left-sided colon cancer: differences in epidemiology, perioperative course, histology, and survival. Dis Colon Rectum 53: 57-64, 2010.

34. Hansen IO and Jess P: Possible better long-term survival in left versus right-sided colon cancer - a systematic review. Dan Med J 59: A4444, 2012.

35. Ito Y, Nakayama T, Miyashiro I, et al: Trends in 'cure' fraction from colorectal cancer by age and tumour stage between 1975 and 2000, using population-based data, Osaka, Japan. Jpn J Clin Oncol 42: 974-983, 2012.

36. Nawa T, Kato J, Kawamoto H, et al: Differences between rightand left-sided colon cancer in patient characteristics, cancer morphology and histology. J Gastroenterol Hepatol 23: 418-423, 2008.

37. Verhulst J, Ferdinande L, Demetter P and Ceelen W: Mucinous subtype as prognostic factor in colorectal cancer: a systematic review and meta-analysis. J Clin Pathol 65: 381-388, 2012.

38. Kawabata Y, Tomita N, Monden T, et al: Molecular characteristics of poorly differentiated adenocarcinoma and signet-ring-cell carcinoma of colorectum. Int J Cancer 84: 33-38, 1999.

39. Kanazawa T, Watanabe T, Kazama S, Tada T, Koketsu S and Nagawa H: Poorly differentiated adenocarcinoma and mucinous carcinoma of the colon and rectum show higher rates of loss of heterozygosity and loss of E-cadherin expression due to methylation of promoter region. Int J Cancer 102: 225-229, 2002.

40. Vogelstein B, Fearon ER, Hamilton SR, et al: Genetic alterations during colorectal-tumor development. N Engl J Med 319: $525-532,1988$. 